\title{
Del desarrollo de capacidades de aprendizaje a la satisfacción del cliente en una instalación hotelera. \\ Caso Hotel Tryp Cayo Coco, destino Jardines del Rey, Cuba.
}

\author{
Edianny Carballo \\ Elme Carballo \\ Raciel Yera \\ Centro de Estudios de Gestión Organizacional y Turismo \\ Facultad de Ciencia Económicas \\ Universidad Ciego de Ávila. \\ Cuba \\ edianny@turismo.unica.cu
}

\section{RESUMEN}

La adecuada ordenación de los sistemas de aprendizaje y su alineación con los objetivos de la organización, requiere de un esfuerzo materializado en la introducción de criterios para decidir cuál es el conocimiento relevante que la organización debe poseer e identificar el que no está disponible en ella para incorporarlo y emplearlo allí donde sea requerido. En tal caso, poner los conocimientos y la innovación en función de un ágil e inteligente desempeño del personal, constituye una premisa de toda organización turística, sobre todo, si se aspira a posicionarse en el mercado con elevados índices de satisfacción. En esta investigación se describe, la incidencia del desarrollo de capacidades de aprendizaje en la satisfacción de los clientes externos en el proceso de Alimentos y Bebidas, (A+B) del Hotel Tryp Cayo Coco. Lo anterior se fundamenta teniendo en consideración la relación entre los conocimientos, los procesos de aprendizaje-innovación y los flujos de información en una organización hotelera. La investigación se realiza en una instalación de cuatro estrellas, perteneciente a la cadena Meliá y enclavada en el destino turístico Jardines del Rey, en Cuba. En el estudio, los resultados del desarrollo de capacidades de aprendizaje se expresan en la mejora del desempeño de los clientes internos del proceso en cuestión y en la introducción de nuevos y mejorados productos y servicios para la satisfacción de los clientes externos.

Palabras claves: Capacidad de aprendizaje, Gestión del conocimiento, Innovación, Satisfacción y flujos de información. 


\title{
The development of learning skills \\ to customer satisfaction in a hotel facility. \\ Case Hotel Tryp Cayo Coco, Jardines del Rey destination, Cuba.
}

\author{
Edianny Carballo Cruz, \\ Elme Emelio Carballo Ramo \\ Raciel Yera Toledo \\ Center for Organizational Studies and Tourism Management \\ Faculty of Economics \\ Ciego de Avila University. \\ Cuba \\ edianny@turismo.unica.cu
}

\begin{abstract}
Proper management of learning systems and their alignment with organizational objectives, it requires an effort materialized in the introduction of criteria for deciding what is relevant knowledge that the organization must identify that it is not available for incorporate it and use it wherever required. In this case, putting knowledge and innovation based on an agile and intelligent performance of staff, is a premise of tourist organization, especially if they are to be positioned in the market with high levels of satisfaction. This research describes the impact of the development of learning capabilities in the external customer satisfaction in the process of Food and Beverage $(A+B)$ of the Hotel Tryp Cayo Coco. This is based taking into consideration the relationship between knowledge, learning processes, innovation and information flows in a hotel organization. Research is conducted on an installation of four-star hotel, the Melia chain and located in the Jardines del Rey tourist destination in Cuba. In the study, the results of the development of learning capacities are expressed in improving the performance of the internal customers of the processes involved and the introduction of new and improved products and services to the satisfaction of external customers.
\end{abstract}

Keywords: learning ability, knowledge management, innovation, satisfaction and information flows 


\section{INTRODUCCIÓN.}

En un contexto caracterizado por la intensa competencia entre los destinos turísticos, resulta cardinal atraer y mantener flujos turísticos crecientes sin sobrepasar por supuesto, la capacidad de asimilación y garantizarles niveles de satisfacción acorde con sus expectativas (Matos, 2005). En otras palabras, cada destino o negocio turístico debe concebirse y desarrollarse con una orientación hacia las necesidades, en constante dinámica y desarrollo, de sus clientes actuales o potenciales. Satisfacer al visitante, muchas veces implica replantearse las "prácticas" o rutinas de trabajo, requiere que las organizaciones sean ágiles, capaces de cuestionar su pasado y de hacer las cosas de forma diferenciada. En estas condiciones, el conocimiento es la clave para mantener la actitud abierta hacia el cambio y hacia una mejora constante.

En consonancia con lo expuesto, al surgir a mediados de los años noventa las organizaciones hipertexto (Nonaka y Takeuchi, 1995; Rodríguez et al. 2001), el turismo da los primeros pasos en la incorporación del proceso de aprendizaje a la estructura organizativa, adaptándola a las necesidades de los clientes sin romper radicalmente con la estructura existente.

Las organizaciones no pueden confiar exclusivamente en el mantenimiento de sus conocimientos y habilidades actuales, sino que deben ser capaces de desarrollar y aplicar nuevos conocimientos, completando y conservando la capacidad de ajuste de sus características internas - productos, servicios, procesos productivos- a las transformaciones del entorno. Todo ello es el fundamento para la existencia de un personal más competente y para la obtención de resultados superiores (Leonard-Barton, 1995). En tal sentido es preciso señalar, que muy ligado al proceso de aprendizaje del licenciado en turismo o del trabajador del sector de forma general, debe situarse al uso del conocimiento para maximizar el valor de la organización, es decir, exhortar al personal a innovar y adaptarse al cambio, Valhondo (2003: 85).

Resulta interesante observar que en los últimos tiempos se está generando una dinámica orientada a fomentar la capacidad de aprendizaje en la organización, como uno de los elementos críticos para mantener a la organización en un estado de desarrollo y evolución permanentes. De hecho, la experiencia muestra claramente como aquellas organizaciones que no han desarrollado su capacidad de aprendizaje han visto reducida su capacidad competitiva; incluso se ha reconocido que el aprendizaje es un comportamiento inherente a toda organización, destinado a mejorar su capacidad de adaptación y anticipación a las exigencias del entorno (Duncan y Weiss, 1979; Dibella et al., 1996), así como a dotarla de una idiosincrasia propia y difícil de imitar fuera de su contexto. Por tanto, desarrollar capacidades de aprendizaje implica, según estos autores, diferenciarse en el mercado y posicionarse como empresas ágiles en entornos competitivos que evolucionan cada vez más en función de las necesidades del cliente y la satisfacción de estas. Cuanto más rápido se esté en condiciones de aprender e incorporar las nuevas y mejoradas prácticas o rutinas organizacionales, más efectivo se será en la captación y complacencia de una estable corriente de visitantes, sobre todo, si tenemos en cuenta que la satisfacción de esta es 
importante para predecir y comprender mejor las respuestas del individuo después de la experiencia de consumo, (Rodríguez y San Martín, 2008).

El objetivo de la presente investigación es describir la incidencia del desarrollo de capacidades de aprendizaje en la satisfacción de los clientes externos. El estudio se desarrolla en el Hotel Tryp Cayo Coco, instalación con modalidad de servicio Todo Incluido y localizado en unas de las zonas ecológicas más importantes del país, en el Archipiélago Jardines del Rey al norte de la provincia de Ciego de Ávila, Cuba. Este hotel, con categoría de cuatro estrellas y perteneciente a la Cadena Sol Meliá, está conformado por 13 bloques habitacionales de dos y tres plantas, diferenciados por la combinación de alturas y utilización de escaleras. La instalación se destaca por su alta capacidad habitacional (508 habitaciones) y el beneficioso flujo de turistas que han sido motivados a conocerlo como producto.

La incidencia del desarrollo de capacidades de aprendizaje en la satisfacción de los clientes externos, se analiza fundamentalmente en el área de Alimentos y Bebidas, $(A+B)$. Este proceso clave integra la cadena del valor de la organización, de ahí que su elección esté motivada por su naturaleza de añadir valor al cliente e incidir directamente en la satisfacción del mismo.

En la actualidad en el Hotel se brinda a los clientes ofertas gastronómicas de distintas tipologías y variedades; cuenta con un restaurante buffet, 7 restaurantes especializados en comida internacional y 5 bares temáticos.

El presente trabajo está estructurado de la siguiente manera: inicialmente se hace referencia a las consideraciones teóricas, donde se describe el enfoque estratégico de la gestión del conocimiento en el desarrollo de capacidades de aprendizaje, y se fundamenta la interacción dinámica entre los conocimientos-los procesos de aprendizaje-innovación y los flujos de información para el incremento de la satisfacción de los clientes externos. En un segundo momento se detalla la metodología a seguir para cumplir el objetivo trazado en la investigación y por último se exponen los resultados, seguidos de las conclusiones y las referencias bibliográficas.

\section{CONSIDERACIONES TEÓRICAS.}

La mayor parte de los estudios e investigaciones enfocados hacia esta cuestión definen el aprendizaje por sus efectos, reconociendo que "es una condición para el cambio sostenido en el estado del conocimiento de un individuo o de una organización", cambio que se refiere tanto a las cualidades del conocimiento que se posee, como al grado de maestría con el que se sabe y se puede actuar sobre lo que sabe. Se trata, ciertamente, de una concepción que engloba los componentes cognitivos y de comportamiento vinculados al conocimiento (Duncan y Weiss, 1979; Fiol y Lyles, 1985; Garvin, 1993; Senge et al., 1990; Walsh y Ungson 1991; Crossan et al., 1999; Rant, 2001; Prieto, 2003: 24; entre otros), según la cual el aprendizaje en la organización representa la transformación tanto del modo de pensar las cosas -aspectos cognitivos del conocimiento-, como del modo de hacer las cosas - 
aspectos relacionados con el comportamiento-. Consecuentemente, según Prieto (2003: 24), el resultado inmediato debe ser un conocimiento nuevo o mejorado.

Para poder definir y valorar la capacidad de aprendizaje del sistema organizativo, se entiende que el paso preliminar y obligado es la disociación del conocimiento en sus dos componentes (Vera y Crossan, 2000): los stocks y los flujos de información y conocimiento, representativos de los aspectos estáticos y dinámicos de los sistemas de aprendizaje. Por consiguiente, las dos dimensiones del conocimiento, estático y dinámico, convenientemente armonizadas constituyen una representación del potencial de aprendizaje superior a la que se obtendría de cualquiera de ellas por separado (Dragonetti y Roos, 1998).

La integración de ambos enfoques es un aspecto fundamental que hace de la gestión del conocimiento una herramienta estratégica adaptada a las características del entorno específico de cada organización (Stankeviviute, 2001). Desde éste punto de vista, la literatura relacionada se ha enfocado sobre la competitividad de la organización, de tal forma que el aprendizaje y los conocimientos son juzgados en función de su valor estratégico. Tal y como señalan Hamel y Prahalad (1993: 74) "no es suficiente con llegar a ser una organización que aprende; una organización debe ser capaz de aprender más eficientemente que sus competidores".

\section{1. El enfoque estratégico de la gestión del conocimiento en el desarrollo de capacidades de aprendizaje.}

La necesidad de relacionar el aprendizaje en la organización con su estrategia, ha estado reconocida durante años por la literatura (Prieto, 2003: 94). El aprendizaje organizacional debe constituir una elección estratégica en una instalación hotelera, como mismo lo constituye la necesidad de satisfacer al cliente para mantenerse vivos en el mercado. Tal es así, que la estrategia de la organización tiene que representar el esfuerzo por anular la brecha estratégica existente entre lo que la organización debe hacer para mejorar su posición competitiva y lo que actualmente está haciendo para competir. Esta brecha estratégica implica, al mismo tiempo, la existencia de una brecha de conocimiento potencial, siempre que exista una diferencia entre los conocimientos necesarios para implantar la estrategia y los que realmente posee la organización conducidos por la movilización de los componentes o recursos del capital humano, estructural, relacional y emocional, hasta llegar a innovar en busca de mayor competitividad (Figura 1).

Por consiguiente, para dar al aprendizaje en la organización un objetivo estratégico, las iniciativas de aprendizaje deben estar dirigidas a cerrar esta carencia estratégica de conocimientos. De esta forma, el éxito de la organización queda condicionado por su talento para alinear su estrategia competitiva con su cartera de conocimientos y para desarrollar el conocimiento necesario para implantar la estrategia deseada (Zack, 1999a). El vínculo existente entre los conocimientos de la organización y su estrategia competitiva queda reflejado en su "estrategia de conocimiento" (Zack, 1999a; Bierley y Daley, 2002) o "estrategia de aprendizaje" (Leavy, 1998). 


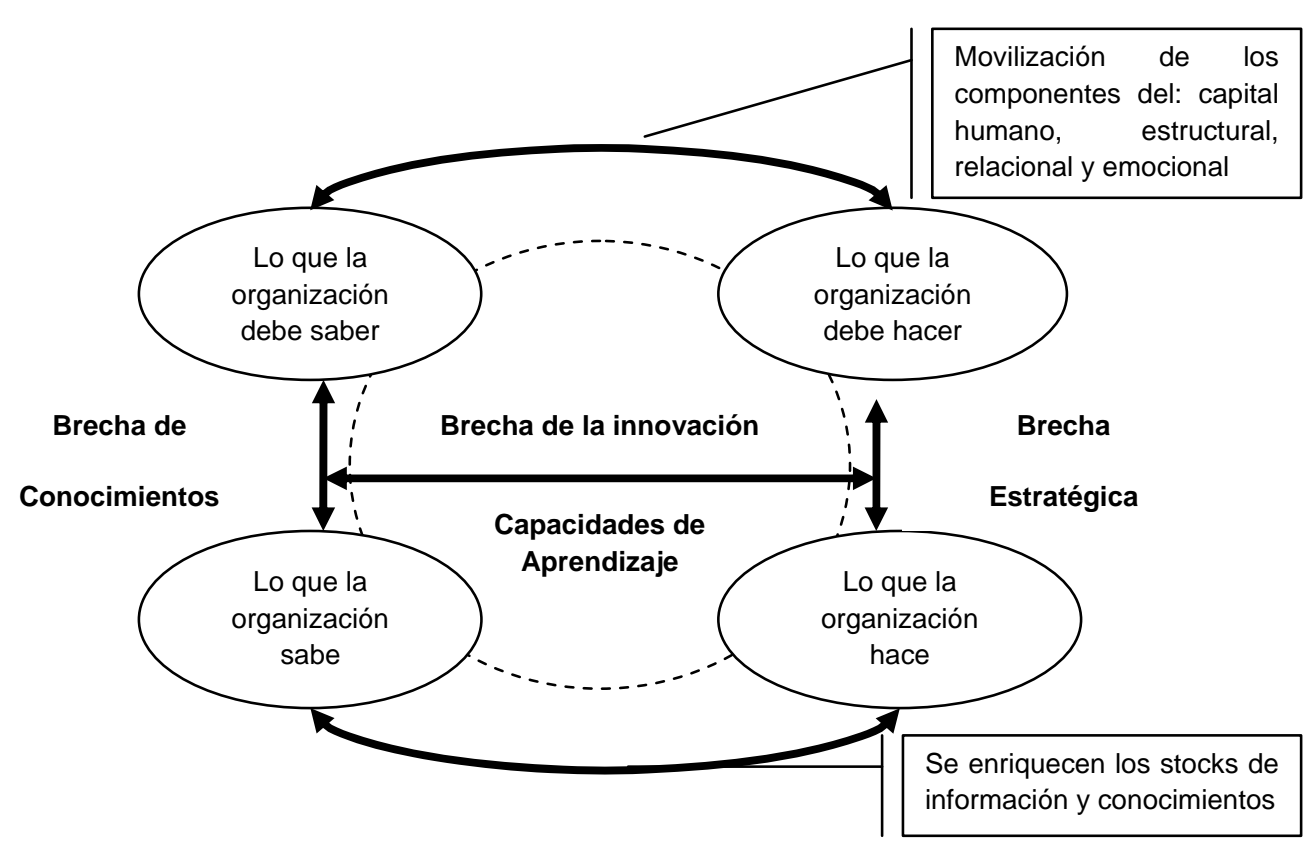

Figura 1: Alineación entre la estrategia y el conocimiento en la organización. Fuente: Zack (1999), asumida y adaptada por los autores.

En este sentido, analizando la satisfacción del consumidor como una cuestión estratégica, es preciso dirigir la conducta de gestión del conocimiento hacia la comprensión y apropiación de las variables que integran el llamado proceso de satisfacción, (Rodríguez y San Martín, 2008: 123). En este caso, se requiere de una exploración profunda de varios conceptos psicológicos -actitudes, procesos de toma de decisiones, emociones, experiencia y satisfacción o lealtad, entre otros- para comprender la psicología del cliente (Crouch et al., 2004) y finalmente disponer la innovación en función de las necesidades de este.

Así pues, según Prieto (2003: 94) para cualquier organización el conocimiento es la base de su competitividad y la gestión de los stocks y los flujos de éste, constituye un elemento moderador de ese vínculo entre el conocimiento y la posición competitiva de la organización. Todas las organizaciones necesitan combinar y alinear los medios técnicos y estructurales con los aspectos más relacionales, intuitivos o sociales como fuentes potenciales de valor a fin de favorecer su capacidad de aprendizaje e innovación.

\subsection{Interacción dinámica entre los conocimientos-los procesos de aprendizaje-innovación y los flujos de información para el incremento de la satisfacción de los clientes externos.}

La gestión del conocimiento está implícita en gestión de la innovación; pero hay que considerar que existe una brecha entre ambas que es necesario abordar, ya que no siempre se gestiona conocimiento para culminar en una innovación. En los servicios en particular, con mucha frecuencia es necesario intercambiar información, experiencias, lecciones aprendidas, impresiones, intuiciones e incluso razonamientos propios que enriquecen el desempeño; pero en ocasiones es un tanto difícil predecir el alcance de su aplicación, sobre todo, cuando 
transciende a individuos, a pequeños grupos, etc; siendo esos casos, particularmente, cuestiones que distan de la innovación, pues no se trata de conocimiento sistematizado y aplicado, traducido en nuevos y/o mejorados productos y servicios.

Por otro lado la forma más genuina de hacer gestión del conocimiento es la capacitación y ¿cuánto no hay que recorrer para lograr que los conocimientos recibidos y adquiridos puedan expresarse en innovaciones? En la figura $\mathbf{2}$ se grafican las relaciones.

Figura 2. Interacción dinámica entre los conocimientos-los procesos de aprendizaje-innovación y los flujos de información para el incremento de la satisfacción de los clientes externos.

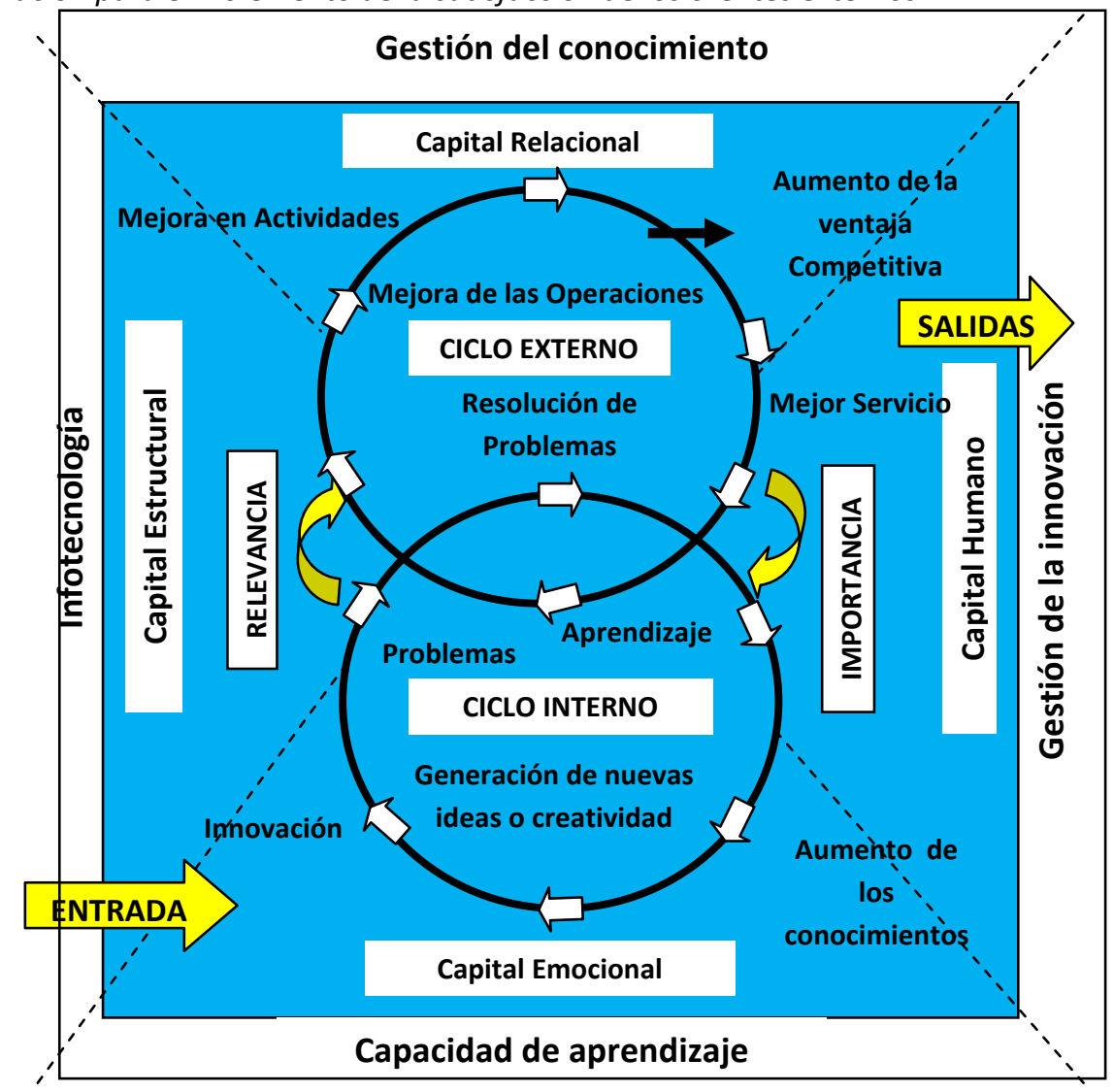

Fuente: Elaboración propia

Específicamente en el Hotel Tryp Cayo Coco, al hacer alusión al ciclo interno se hace referencia a la gestión del proceso de Alimentos y Bebidas, que necesariamente tiene que transitar por la formación de un equipo o colectivo, que de algún modo se conecta en lo cognitivo y expresa su inteligencia emocional en el trabajo (Martínez, 2008). Esto último favorece la determinación y correcta disposición de los roles laborales durante la prestación del servicio; así como la eficacia de la estructura del proceso y de las interrelaciones entre los miembros de este.

Las interrelaciones antes mencionadas, son definidas por los investigadores como "el sistema de disposiciones interpersonales, orientaciones y expectativas devenidas de las valoraciones y emociones en el proceso de comunicación interna del colectivo durante su 
desempeño". La concepción anterior, exige obligatoriamente la existencia de mecanismos de integración y coordinación de distintas tipologías, (formales, informales, estructurados y no estructurados, etc). Dichos mecanismos deben estar orientados sobre la base de crear relaciones ante todo movidas, por el contenido socialmente valioso y significativo para la actividad conjunta. Este pensamiento aboga también, por compartir información y conocimiento para forjar las capacidades de aprendizaje y desarrollar la innovación en la propia gestión del proceso de A+B.

Es imprescindible que en la trayectoria que va desde la identificación de problemas hasta la innovación, en el momento mismo de la resolución de las dificultades, se tenga que estar en contacto con el ciclo externo y en particular con los clientes. Esto implica clasificarlos por segmento de mercado; contemplándose aspectos como la edad, el sexo, las preferencias, hábitos de alimentación, costumbres, cultura, entre otros. En este caso, Decrop y Snelders (2005) argumentan que las tipologías turísticas no deberían considerarse universales y que la influencia del entorno cultural debe tomarse en cuenta para complacer sus exigencias. Naturalmente, la información que se obtenga del cliente externo engrosa los stocks y los flujos de información y conocimiento, posibilitando tomar decisiones a partir de las tecnologías e insumos disponibles, que sin duda, obligan a un permanente desarrollo en cuanto a las capacidades de aprendizaje, generando nuevas ideas, que necesariamente fertilizan el pensamiento creativo para finalmente, innovar.

El punto de contacto del ciclo interno con el externo, no sólo está sujeto a la resolución de problemas, sino que en la interconexión con este último es donde se cultiva la mejora de actividades y operaciones para un mejor servicio. Esto conduce a una ventaja competitiva, a través de un proceso innovativo permanente que interactúa con la infotecnología-gestión del conocimiento y las capacidades de aprendizaje en un escenario donde necesariamente, se integran y movilizan el capital humano, capital estructural, capital relacional y el capital emocional.

En estas relaciones entre ciclo interno y externo, se articula y producen interacciones en una unidad dialéctica, que facilitan la adecuada dinámica de la gestión por procesos durante la prestación de servicio. En consecuencia, en la medida en que los clientes internos tienen un buen desempeño y desarrollan sus capacidades de aprendizaje desde la gestión del conocimiento, también fortalecen la relevancia del servicio. De igual forma, al aumentar satisfacción de los clientes externos con la innovación crece la importancia del servicio recibido y la competitividad de la organización.

\section{METOdOLOGÍA}

Como se ha explicado previamente, el objetivo de este trabajo es describir la incidencia del desarrollo de capacidades de aprendizaje en la satisfacción de los clientes externos en el proceso de A+B del Hotel Tryp Cayo Coco. Para alcanzar dicho propósito, participan en el estudio la totalidad de los clientes internos del proceso mencionado, (Tabla 1): 
Tabla 1. Trabajadores del proceso de A+B del hotel Tryp Cayo Coco.

\begin{tabular}{|l|l|l|l|l|}
\hline \multirow{2}{*}{} & \multicolumn{4}{l|}{ Cantidad de trabajadores según nivel de ocupación (A+B) } \\
\cline { 2 - 5 } & $\mathbf{1 0 0 \%}$ & $\mathbf{8 0 \%}$ & $\mathbf{6 0 \%}$ & $\mathbf{4 0 \%}$ \\
\hline Subdirector de A+B & 1 & 1 & 1 & $\mathbf{1}$ \\
\hline Especialistas de A+B & 2 & 2 & 2 & $\mathbf{2}$ \\
\hline Jefe de Bares & 1 & 1 & 1 & $\mathbf{1}$ \\
\hline Jefe de Restaurantes & 1 & 1 & 1 & $\mathbf{1}$ \\
\hline Capitanes & 12 & 12 & 12 & $\mathbf{1 2}$ \\
\hline Cubre capitanes & 2 & 2 & 2 & $\mathbf{2}$ \\
\hline Dependientes & 70 & 66 & 54 & $\mathbf{4 6}$ \\
\hline Cubre dependientes & $\mathbf{1 2}$ & $\mathbf{1 1}$ & $\mathbf{9}$ & $\mathbf{8}$ \\
\hline
\end{tabular}

Fuente: Elaboración propia

Por otro lado, se tienen en consideración el criterio de 56.638 clientes externos de los principales mercados emisores del Hotel Tryp Cayo Coco, (6.706 clientes de Reino Unido y 49.932 de Canadá). Esta cifra constituye el total de visitantes de estos mercados en el período de realización del estudio, (enero 2009 a diciembre 2010).

Se impone acotar que en el 2010, de los 37.834 clientes recibidos en la instalación, el $72,69 \%$ eran de Canadá y Reino Unido. La representatividad de estos mercados se ilustra también en la cantidad de clientes reincidentes o repitentes, puesto que más del $95 \%$ de ellos provienen precisamente de estos territorios.

Es preciso señalar que se trata de una muestra intencional u opinática (Latorre et al., 2003) y en este caso, según Lohr, (1999: 2-3), se impone delimitar que dentro de las muestras intencionadas existen "elementos típicos" o "representativos" a los que ya públicamente se les ha reconocido tal rango. En este estudio participan como parte del grupo de trabajo, 15 clientes reincidentes de Reino Unido y Canadá. Estos clientes, tienen un promedio de más de 10 visitas al Hotel en los últimos 5 años, por tanto sus percepciones sobre los productos, servicios y competencias de los trabajadores son más amplias que la de otros visitantes. Las razones anteriores justifican su reconocimiento y selección, como "elementos representativos" y participantes directos en el estudio.

El uso de los métodos de investigación empírica, se expresan en la realización de una serie de procedimientos prácticos con el objeto y los medios de investigación, permitiendo revelar las características fundamentales y relaciones esenciales del primero. Se emplean el método de la observación científica, la encuesta, las entrevistas grupales e individuales y la medición.

La opinión de los clientes internos se recoge fundamentalmente a través de las guías de observación, la encuesta sobre creación y organización del conocimiento y las entrevistas grupales e individuales. La de los clientes externos se obtiene mediante las guías de observación encuestas y las corporativas de la Cadena Sol Meliá. Estas últimas se emplean, junto con el correspondiente sistema informático que soporta la información estadística, para conocer la satisfacción del turista. En dichas encuestas se contemplan todos los productos y servicios del Hotel Tryp Cayo Coco, a través de un cuestionario que capta la 
opinión del turista, relacionada con el desempeño los trabajadores. En el instrumento se puede evaluar a través del check box de Muy Bien, Bien, Mal y Muy Mal los aspectos e indicadores tratados. No se incluyen términos medios, como regular para evitar que el cliente responda de forma automática sin tener un juicio real de la evaluación emitida. En el cuestionario existe un espacio donde el cliente puede añadir comentarios o sugerencias.

Cada aspecto evaluado obtiene una calificación entre 1 a 5 puntos. La fórmula de evaluación que se aplica es:

Puntuación $(\mathrm{P})=(\mathrm{CMB} \times 1+\mathrm{CB} \times 2+\mathrm{CM} \times 4+\mathrm{CMM} \times 5) / \mathrm{CT}$;

Donde CMB- Cantidad de encuestados que evaluaron el aspecto o indicador de Muy Bien (MB);

CB- Cantidad de encuestados que evaluaron el aspecto o indicador de Bien (B);

$\mathrm{CM}$ - Cantidad de encuestados que evaluaron el aspecto o indicador de Mal (M);

CMM- Cantidad de encuestados que evaluaron el aspecto o indicador de Muy Mal (MM);

CT- Cantidad Total de encuestados.

La tendencia a 1 es una mejoría de los aspectos e indicadores evaluados. Los intervalos establecidos para la clasificación de la puntuación se recogen en la siguiente tabla:

Tabla 2. Clasificación de los indicadores de Calidad. Fuente: Cadena Sol Meliá, Cuba.

\begin{tabular}{|l|l|}
\hline Clasificación & Puntuación \\
\hline Seguro que tiene sentido & $\leq 1.43$ \\
\hline Tiene sentido & $1.44-1.55$ \\
\hline Tendrá sentido & $1.56-1.76$ \\
\hline
\end{tabular}

Fuente: Elaboración propia

Por otro lado, es necesario acotar que el aporte final de este trabajo está dado por el desarrollo de capacidades de aprendizaje, expresado en términos de un mejor desempeño de los clientes internos y en el diseño de nuevos y/o mejorados productos y servicios para la satisfacción de los clientes externos, lo que finalmente implica la ocurrencia de un impacto organizativo positivo.

Figura 3. Impacto organizativo del desarrollo de capacidades de aprendizaje en el proceso de A+B del Hotel Tryp Cayo Coco.

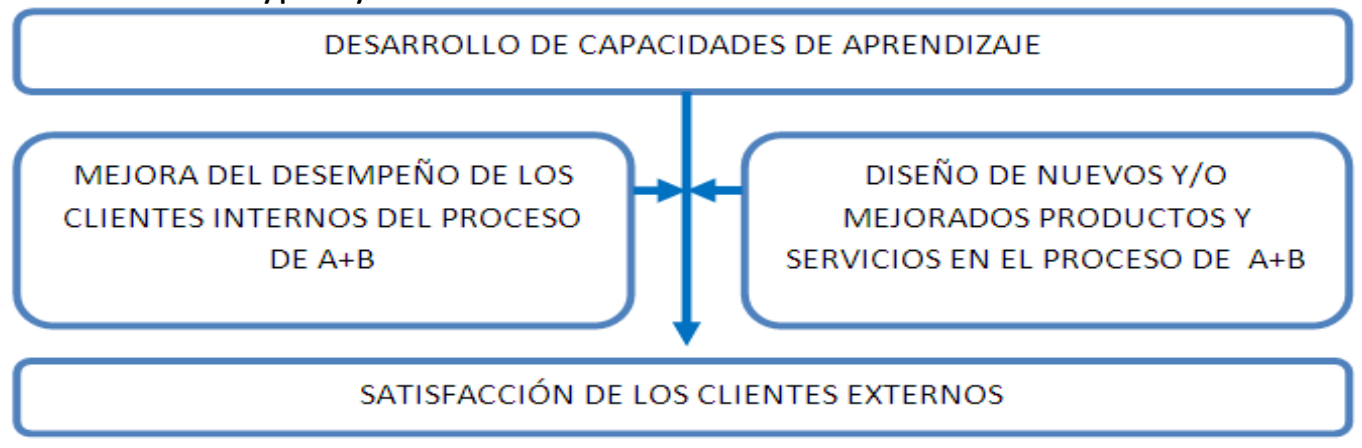

Fuente: Elaboración propia

Investigaciones Turísticas. 


\section{MOVILIZACIÓN DE CONOCIMIENTOS PARA SATISFACER AL CLIENTE.}

\subsection{Percepciones de los clientes internos en el proceso de $A+B$.}

En el intercambio sostenido con los trabajadores y directivos, del proceso de $A+B$ se realizan entrevistas grupales e individuales para diagnosticar el estado de la gestión del conocimiento y la innovación en la organización en pos del desarrollo de capacidad de aprendizaje para la satisfacción de los clientes externos.

En este sentido, se obtiene que el $80 \%$ de los participantes tiene una concepción limitada de la frase "gestión del conocimiento", esta es concebida como sinónimo de capacitación. En esta parte, los capitanes y cantineros de bares manifiestan su inconformidad con los escasos cursos que les imparten y los temas que allí se tratan, opinan además, que la capacitación puede ayudarlos a diferenciar el servicio y satisfacer al cliente. Los procesos de generación y transferencia del conocimiento, generalmente no ocupan parte del pensamiento de los actores involucrados, la preocupación se centra en la adquisición de conocimientos, específicamente en actividades formales y menos aún en compartir lo aprendido. En las organizaciones cubanas del Turismo la generación de conocimientos está asociada fundamentalmente al desarrollo de proyectos de investigación universitarios y centros de investigación, mientras que con el sistema ESCUELA DE HOTELERÍA Y TURISMOUNIVERSIDAD en la preparación de los trabajadores, técnicos y directivos se han abierto otras expectativas que bien pudieran asociarse a la solución de los problemas propios de las instituciones del turismo.

Por otro lado, al hacer referencia, a la innovación, el 100\% de los directivos plantean que en la organización existe un compromiso con esta y que ellos se muestran abiertos a las iniciativas, exigencias e ideas de los trabajadores; sin embargo el 50\% de los dependientes de servicio gastronómico no piensa igual. Se asume también por parte de estos últimos, a la innovación como sinónimo de creatividad.

\subsubsection{Patrones de aprendizaje de los clientes internos.}

Las entrevistas realizadas a nivel individual y colectivo, además de la observación, muestran que los clientes internos del proceso de bares en el Hotel Tryp Cayo Coco se limitan a ser recolectores de datos e información en los intercambios informales y en las experiencias extraídas del quehacer cotidiano, sobre todo, en el contacto con los clientes. La adquisición de conocimientos es principalmente a través de la búsqueda individual, en menor cuantía por la asistencia a los cursos de la temporada baja y menos aún, por la participación en eventos, por la asistencia a formas de capacitación y superación postgraduada y por el uso de fuentes documentales (revistas especializadas, Internet, normativas corporativas, etc.). En la transferencia del conocimiento predominan los encuentros informales y la voluntad de los actores involucrados para intercambiar conocimientos y experiencias. 


\subsection{Facilitadores de creación de conocimiento en el desarrollo de capacidades de aprendizaje.}

Para valorar cómo se comporta la creación y organización del conocimiento en la organización estudiada y su utilidad en el desarrollo de capacidades de aprendizaje se aplica un cuestionario a los clientes internos compuesto por varios bloques, (Datos de clasificación, Diseño organizativo, Facilitadores de la creación de conocimiento y Creación de conocimiento). Dentro de estos, aparecen afirmaciones para ser valoradas entre 1-10 puntos, según el criterio del encuestado, donde 1 es Totalmente en desacuerdo y 10 Totalmente de acuerdo.

En este estudio, los autores se centran en determinar el comportamiento de los facilitadores de conocimiento para el desarrollo de capacidades de aprendizaje. Este apartado cuenta con 22 ítems que se agrupan en las afirmaciones que se detallan a continuación. En este trabajo no se incluyen todos los ítems del cuestionario; solamente aquellos que ilustran con más claridad los resultados obtenidos.

Del 80 al 100\% de los clientes internos están de acuerdo con las siguientes afirmaciones:

- Cuando se dota a los equipos y al personal de autonomía, esto les proporciona la motivación suficiente para crear, aplicar y absorber nuevo conocimiento.

- La ruptura de hábitos y rutinas de trabajo y la introducción de desafíos de manera intencionada motiva a los individuos y equipos a superarse.

- Es más importante, cambiar y retar al entorno que adaptarse.

- Habitualmente, se llevan a cabo reuniones para compartir conocimientos e ideas y discutir asuntos de trabajo.

- Los equipos o unidades destinadas al desarrollo de un nuevo producto, mantienen durante esta actividad contacto con el entorno.

- La estructura de gestión y organización de la empresa, está preparada para tratar con la complejidad del entorno, sin embargo a veces están apegados a los estándares de la Cadena Sol Meliá.

- Es frecuente en la organización la reestructuración y modificación de la estructura organizativa para enfrentarse a problemas o situaciones diversas.

- La cooperación y la confianza mutua son valores que aparecen explícitamente recogidos en los objetivos de la empresa y son compartidos por la mayoría de sus miembros.

Por otro lado hay desacuerdo con las aseveraciones siguientes:

- Una vez explicadas las guías de actuación o estrategia de la empresa, se provee a los equipos de independencia en la toma de decisiones relacionada con sus tareas.

- Se intenta, de una manera intencionada, transmitir tensión evocando un sentido de crisis proponiendo situaciones desafiantes a resolver.

- Se impulsa a competir a diferentes equipos en el desarrollo de un nuevo producto o proyecto. 
- Existen programas de rotación de personal entre las distintas áreas funcionales de la empresa.

- Las diferentes áreas funcionales trabajan de forma solapada para el desarrollo de un producto o proyecto nuevo.

Con estos resultados se evidencia el compromiso de la organización con la movilización del conocimiento en pos de la preparación de sus miembros; sin embargo, se comprueban limitaciones fundamentalmente, en lo que se refiere a compartir lo aprendido y a gestionar el conocimiento en la organización para innovar. Estos resultados y sobre todo el limitado conocimiento del alcance de la innovación, se corresponden con los obtenidos las entrevistas. En estas últimas más del $50 \%$ de los entrevistados asocia a la innovación con las necesidades de satisfacer al cliente y obtener propina, agregando además que esta se utiliza para mitigar la falta de recursos, es decir, "hacer más con menos".

Lo anterior evidencia la necesidad de emprender acciones o dictar políticas organizacionales, para eliminar las barreras que dificultan la gestión del conocimiento y la innovación y con ello, contribuir al desarrollo de capacidades de aprendizaje que lleven a un mejor desempeño y por tanto a la satisfacción de los clientes externos en el área que se analiza.

\subsection{Actividades para un mejor desempeño de los clientes internos en el proceso de $A+B$ del Hotel Tryp Cayo Coco.}

Como un elemento determinante, durante la marcha de la investigación, se capacita a los participantes en el estudio a través de talleres y de acciones programadas con el departamento de Recursos Humanos, la Escuela de Hotelería y Turismo (Formatur) y la Universidad de Ciego de Ávila. Las principales actividades desempeñadas se destacan a continuación:

1. Se realiza una conferencia - taller, en el mes de diciembre de 2009, sobre los Impactos y la relación de la formación e investigación en el Hotel Tryp Cayo Coco. En esta actividad participan profesores de la Universidad de Ciego de Ávila y de Formatur. Tienen acceso, además los trabajadores y directivos del proceso de A+B, los directivos de RR. HH, calidad, atención al cliente, alojamiento, etc.

2. Se crean y aprueban las Estructuras Paralelas de Aprendizaje (EPA) con más de 20 intercambios dentro y fuera de la instalación. Es una estructura creada para el aprendizaje, la innovación y la creación de conocimiento que suplementa, no reemplaza, a la organización principal. Tiene un carácter temporal y actualmente la preside el Subdirector de A+B de la instalación.

3. Se crean tres Equipos de trabajo, dos comprenden el turno de trabajo diurno y nocturno del Restaurante Buffet "Los Taguascos" y el tercero incluye a los capitanes y dependientes del Lobby Bar "El Manglar" y del Snack Bar "Las Antillas". Estos equipos son responsables del trabajo regular y continuo de la organización en materia de vigilancia de las necesidades de los clientes ingleses y canadienses y en función de la introducción de nuevos y/o mejorados productos. Tienen un carácter permanente. 
4. El coordinador de capacitación nacional de la Asociación de Cantineros de Cuba, visita la instalación e imparte 3 cursos de enero a marzo de 2010, sobre las Tendencias de los servicios de bares. En estos cursos participa un representante por área y socializa sus conocimientos en las Reuniones de Información correspondientes.

5. Se establece un programa de rotación en los puestos de trabajo. En el mes de noviembre del 2010 habían rotado el 51\% de los capitanes y cantineros del área.

6. Los participantes en el estudio nombran, a los expertos del proceso de A+B del Hotel Tryp Cayo Coco y a dos consultores externos de la Asociación Nacional de Cantineros de Cuba. La selección de los expertos estuvo avalada por la experiencia laboral, los cursos recibidos, reconocimientos, etc. La función de dichos especialistas es capacitar a los demás trabajadores del área y evaluar la introducción de nuevos y/o mejorados productos y servicios.

7. Se diseña un Manual de Términos para el Turismo que incluye otras áreas del saber asociadas al sector. Éste consta de más de 2000 términos, incluyendo además "mejores prácticas" y procedimientos. De igual manera con el objetivo de disponer de un stock de información actual asociado al área A+B, el Manual en una de sus partes contiene un Glosario de Términos Culinarios que recoge un amplio vocabulario relacionado con la cocina y el servicio de salón, además de un recetario de cócteles y comidas nacionales e internacionales. En este apartado se listan y caracterizan, igualmente, las enfermedades digestivas que pueden afectar a los clientes durante su estancia; detallándose los alimentos más frecuentemente contaminados, el período de incubación, la sintomatología y el tratamiento. Este documento, en formato digital, se encuentra a disposición de los dependientes gastronómicos, directivos del área y está diseminado en las instituciones regionales relacionadas con el Turismo y en todas las Escuelas de Hotelería y Turismo del país.

\subsection{Introducción de nuevos y mejorados productos en el proceso de $A+B$.}

De las prácticas e iniciativas de gestión de conocimientos (capacitación, creación, socialización y transferencia de conocimientos, etc.) y de las acciones desarrolladas con los directivos, capitanes y dependientes del área de Alimentos y Bebidas surgen nuevos y/o mejorados productos, servicios y actividades en correspondencia con las exigencias $y$ necesidades de los clientes externos. Lo anterior demuestra una mejoría en la preparación y en el desempeño del personal.

En este sentido en el restaurante buffet "Los Taguascos" se incorpora durante del desayuno en una sección de alimentos dietéticos integrados por cereales, mermeladas, panes, galletas, etc. Productos estos, demandados por el $40 \%$ de los clientes de Canadá y Reino Unido.

Por otro lado, se diseña un menú digital, al que pueden acceder los consumidores, cuando estén en el Cybercafé. Éste está formado por una lista de cocteles, los ingredientes de los mismos, los tipos de café y otras bebidas que se ofertan en el Lobby Bar. Es importante señalar que el menú digital se realiza teniendo en cuenta el criterio de los directivos, capitanes y cantineros de bares y las preferencias de los consumidores detectadas 
durante la investigación. Desde él se puede enviar, mediante una opción de correo habilitada en la página, sugerencias relacionadas con el servicio directamente a la oficina de Atención al Cliente.

De igual forma se describen brevemente, en el menú digital o página web, los bares de la instalación, su tipología, horarios, etc.

\subsubsection{Estilos del servicio de café.}

Se introducen siete estilos de presentación del tradicional café con leche (Flor, Mitad \& Mitad, Amanecer, Corazón, Árbol, Hoja) o también llamado capuchino, cortadito, en dependencia de la disminución o aumento de uno de los componentes base. En este sentido se trabaja con los siguientes ingredientes: café, leche, crema de leche, chocolate líquido y en polvo. Los estilos desarrollados son incluidos en las prestaciones de los bares, (Lobby Bar y Snack Bar), que ofertan servicio de café en el desayuno y después del almuerzo y la cena.

Según el registro llevado a cabo por los capitanes y dependientes, los estilos más demandados por clientes en la última semana del mes de noviembre de 2010 fueron:

- Mujeres de Reino Unido y Canadá: flor, amanecer y mitad \& mitad, (entre 40 y 45 pedidos).

- Hombres de Reino Unido: mitad \& mitad y caracol, (20 pedidos).

- Hombres de Canadá: árbol, amanecer y caracol, (entre 30 y 35 pedidos).

\subsubsection{Nuevos y/o mejorados cocteles}

En el servicio de bares se introducen 14 nuevos y/o mejorados cocteles para el mercado de Reino Unido y Canadá. Las recetas de estos emergen de la creatividad individual de los cantineros y de los intercambios en los talleres realizados durante el estudio. Las propuestas de cocteles se introducen durante las prestaciones y en dependencia de la aceptación por parte de los clientes externos, se institucionalizan como práctica del proceso. En el 35\% de los cocteles que existían con anterioridad en el menú de los bares, el cambio o alteración de sus ingredientes es mínimo; siendo mejorados o transformados en dependencia de las demandas y preferencias del consumidor.

En la tabla 3 se citan dos ejemplos de cocteles renovados (sustitución de uno de sus ingredientes) y dos de cocteles nuevos de gran aceptación entre los clientes. 
Tabla 3. Nuevos y/o mejorados cocteles en el proceso de A+B.

\begin{tabular}{|c|c|c|c|}
\hline \multirow{2}{*}{\multicolumn{2}{|c|}{ Bebida Base Coctel }} & Ingredientes de la receta original & Variación de la receta original \\
\hline & \multicolumn{3}{|c|}{ Cocteles modificados } \\
\hline \multirow{2}{*}{$\begin{array}{lr}\text { Coñac } & \\
\text { Ron } & \text { Havana } \\
\text { Club } & \text { (añejo } \\
\text { blanco) } & \end{array}$} & Banana Mama & $\begin{array}{l}\text { En la coctelera: } \\
\text { Hielo, piña colada, granadina, licor de } \\
\text { banana y ron. }\end{array}$ & $\begin{array}{l}\text { En la batidora: } \\
\text { Hielo, piña colada, granadina, } \\
\text { banana en fruta y ron. }\end{array}$ \\
\hline & $\begin{array}{l}\text { Piña Colada con } \\
\text { licor }\end{array}$ & $\begin{array}{l}\text { En la batidora: } \\
\text { Leche, zumo de piña, crema de coco, } \\
\text { ron, azúcar, hielo. }\end{array}$ & $\begin{array}{l}\text { En la batidora: } \\
\text { Crema de coco, jugo de piña, licor } \\
\text { de piña, ron y hielo. }\end{array}$ \\
\hline \multicolumn{4}{|l|}{ Nuevo coctel } \\
\hline \multirow[t]{2}{*}{$\begin{array}{lr}\text { Ron } & \text { Havana } \\
\text { Club } & \text { (añejo } \\
\text { blanco) } & \end{array}$} & $\begin{array}{l}\text { Festival } \\
\text { Caribeño }\end{array}$ & $\begin{array}{l}\text { En la coctelera: } \\
\text { Azúcar, jugo de limón, vermouth } \\
\text { blanco seco, vermouth rojo, ron, hielo. }\end{array}$ & - \\
\hline & $\begin{array}{l}\text { Blue Coco o } \\
\text { Club Coco }\end{array}$ & $\begin{array}{l}\text { Hielo, jugo de naranja, ron, granadina, } \\
\text { curaçao azul. }\end{array}$ & - \\
\hline
\end{tabular}

Fuente: Elaboración propia

\subsection{Evaluación de la satisfacción de los clientes externos.}

Con el desarrollo de capacidades de aprendizaje y la introducción de nuevos y/o mejorados productos y servicios durante el transcurso de la investigación se logra un incremento de la satisfacción de los clientes externos. Lo expresado se corrobora con el análisis de los indicadores de satisfacción al cliente, teniendo en cuenta dos períodos: el primero antes de desarrollar capacidades de aprendizaje en el proceso de A+B, (enero a diciembre 2009) y el segundo después de aplicar las iniciativas de gestión del conocimiento y la innovación para el desarrollo de dichas capacidades, marcado por la introducción de nuevos y/o mejorados productos y servicios, (enero - noviembre 2010).

Los criterios de los clientes externos de Reino Unido y Canadá son favorables con respecto al desempeño de los trabajadores del proceso que se analiza. En las encuestas corporativas de los meses de octubre y noviembre del 2010, el 51,77\% de los 1.393 clientes encuestados hacían referencia al buen desempeño de un trabajador del área de bares y el $100 \%$ de los 620 encuestados en octubre, calificaba de bien y muy bien la calidad de la oferta, mientras que el $99,68 \%$ otorgaban iguales calificaciones a la variedad de las bebidas. En el mes de noviembre el $100 \%$ de los 773 encuestados, en ambos indicadores, otorgaban calificaciones de bien y muy bien. Por otro lado, los clientes externos destacan la amabilidad, la creatividad de los dependientes y la diferenciación y personalización del servicio, según las manifestaciones registradas a través de la observación no participante.

Como se explica en el apartado III de este artículo, cuando los resultados de los indicadores de satisfacción del cliente tienden a 1, esto representa una mejora de los aspectos evaluados y por tanto de la complacencia del turista. En el caso que ocupa a esta investigación se tiene que la calidad de las comidas y bebidas en el 2009 oscila entre 1,50 y 1,73 mientras que en el 2010 se sitúa entre 1,45 y 1,58 (Gráfico 1). La variedad de las comidas y bebidas se comporta de forma similar; en el 2009 se observa que los resultados fluctúan entre 1,53 y 1,71 y en el 2010 estos oscilan entre 1,43 y 1,59. Los meses de mejores 
resultados en el 2010 fueron mayo, julio y agosto. Coincidiendo con el período de mayor introducción de productos y/o servicios.

Los resultados descritos demuestran, que con la introducción de nuevos y/o mejorados productos se logra una mayor diversidad en el menú por medio de la innovación y combinación de recetas. Es importante señalar en este caso, que lo anterior se alcanza sin alterar lo normado en los estándares de la Cadena Sol Meliá.

Gráfico 1. Evolución de los indicadores de calidad y variedad de las comidas y bebidas en el proceso de A+B del Hotel Tryp Cayo Coco, 2009/2010.

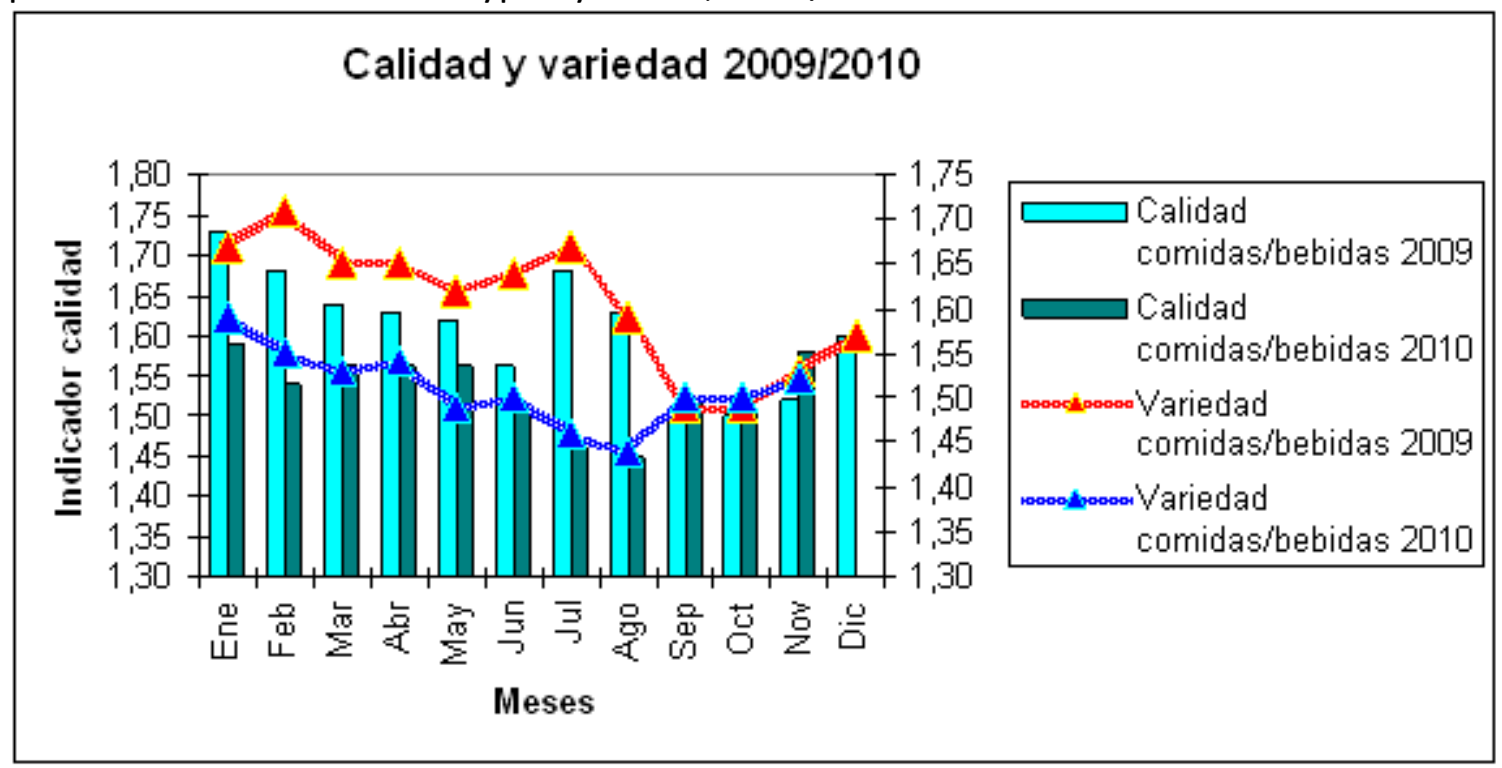

Elaboración propia

Al analizar el comportamiento de los indicadores por bares en el 2009, estos muestran resultados $\leq 1.43$, (seguro que tiene sentido) los siguientes:

- Bar Playa "La Barcaza": 1,38

- Aqua Bar "El Delfín": 1,39

- Lobby Bar "El Manglar": 1,40

En este sentido, en el 2010 se observa que los valores de los bares por meses, tienden más a 1 y que aquellos con mejores indicadores son:

- Aqua Bar "El Delfín": 1,37. Mejora con respecto al 2009.

- Bar Playa "La Barcaza": 1,41

- Bar Discoteca "Vida Loca": 1,37

- Lobby Bar "El Manglar": 1,40

- Bar Playa "La Picúa": 1,43

En el rango de 1,44 -1,55, (tiene sentido) en el 2009 se encontraba el Snack Bar "Las Antillas", con un valor promedio de 1,54. En cambio, en el 2010 se sitúa dentro de este 
rango, igualmente el Snack Bar "Las Antillas", pero con un valor de 1,44, lo que expresa una mejoría con respecto al año precedente.

\section{CONCLUSIONES.}

El presente estudio ha contribuido a determinar cómo incide el desarrollo de capacidades de aprendizaje en la satisfacción de los clientes externos en un proceso clave de una instalación hotelera. Para ello se tuvo en consideración la disociación del conocimiento en dos componentes fundamentales, los stocks y los flujos de información y conocimiento, elementos cuya adecuada identificación y gestión se expresan en el aumento de la sapiencia organizacional y por ende en el mejor desempeño de su capital humano. La investigación denota la necesidad de un enfoque estratégico de gestión del conocimiento, basado sobre todo en la combinación y alineación de los medios técnicos y estructurales por los que fluye el conocimiento con los aspectos más relacionales, intuitivos o sociales para el desarrollo de capacidades de aprendizaje. Para el desarrollo del estudio los investigadores asumen o describen la interacción dinámica entre los conocimientos-los procesos de aprendizajeinnovación y los flujos de información, a través de dos ciclos cuyo punto de contacto implica la mejora de actividades y operaciones para un mejor servicio y el incremento de la satisfacción de los clientes externos.

Toda organización puede ser considerada como un sistema de acciones deliberadas. Éste debe estar comprometido con el proceso de transformación y dirigido a la producción de determinados resultados en su entorno de actividad. En conformidad con esto, este estudio muestra que el óptimo desempeño de los trabajadores en el proceso de A+B nace de las acciones o prácticas e iniciativas de gestión de conocimiento (capacitación, creación, socialización y transferencia de conocimientos, etc.) que a su vez son disparadores de la creatividad e innovación organizacional durante la prestación de servicios.

Los resultados de la investigación demuestran a través de la conducta de los indicadores de satisfacción, que la introducción en el proceso de $A+B$ de nuevos $\mathrm{y} / \mathrm{o}$ mejorados productos y servicios contribuye al incremento de la satisfacción del cliente. Tal es así, que el comportamiento del total de los indicadores en el 2010, después de introducidos los resultados, muestra una mayor tendencia a 1 ; lo que representa un incremento en la complacencia del cliente durante la prestación del servicio. Es innegable que lo anterior no sería posible si no se conocen las demandas y preferencias de los clientes, las "buenas prácticas", tendencias del servicio, etc. De ahí la importancia de gestionar el conocimiento, alimentar los stocks y los flujos de información y conocimiento para tomar decisiones oportunas a partir de las tecnologías e insumos disponibles.

Por otro lado la investigación se ve limitada por el hecho de que se circunscribe al contexto de una instalación hotelera. Para Prieto, (2003: 28) una organización es un sistema de aprendizaje que debe desarrollar su actividad en continua interacción con el entorno con el propósito de interpretarlo y de adecuarse a sus condiciones. La cuestión que esto suscita para comprender el funcionamiento de todo sistema de aprendizaje es la de considerar esta 
confrontación entre la organización y el entorno y analizar su trascendencia en la dinámica de desarrollo de la capacidad de aprendizaje

El desarrollo de capacidades de aprendizaje en otros procesos, (estratégicos o en de apoyo) y por qué no en un destino turístico, podría significar una nueva forma de representarse el aprendizaje organizacional. En este sentido habría que abundar más en los mecanismos de integración y coordinación, en los componentes tangibles e intangibles de la gestión del conocimiento, entre otros.

\section{REFERENCIAS BIBLIOGRÁFICAS.}

BIERLEY, P. Y DALEY, P. (2002). “Aligning Human Resource Management Practices and Knowledge Strategies". En C. Wei Choo y N. Bontis (eds.). The Strategic Management of Intellectual Capital and Organizational Knowledge, Oxford University Press

CROSSAN, M.M., LANE, H.W. Y WHITE, R.E. (1999). "An Organizational Learning Framework: from Intuition to Institution." Academy of Management Review, vol. 24, nㅇ, pág. 522-537.

CROUCH, C., PERDUE, R., TIMMERMANS, H. AND UYSAL, M., (2004). Consumer Psychology of Tourism, Hospitality and Leisure. London: CABI Publishing.

DECROP, A. AND SNELDERS, D., (2005). "A grounded Typology of Vacation Decision-Marking". Tourism Management, vol 26, pág. 121-130.

DIBELLA, A.J., NEVIS, E.C. Y GOULD, J.M. (1996B). "Organization Learning Style as a Core Capability." En Moingeon, B. y Edmondson, A. Organizational Learning and Copetitive Advantage, Sage Publications.

DRAGONETTI, N.C. Y ROSS, G. (1998). "Efficiency and Effectiveness in Government Programmes: An Intellectual Capital Perspective". 2nd World Congress on Intellectual Capital, January 1998, Hamilton, Canada: McMasters University,

DUNCAN, R. Y WEISS, A. (1979): “Organizational Learning: Implications for Organizational Design." Research in Organizational Behaviour, vol.1, pág. 75-123.

FIOL, C.M. Y LYLES, M.A. (1985). “Organizational Learning." Academy of Management Review, vol.10, no4, pág. 803-813.

GARVIN, G.A. (1993). "Building a Learning Organization." Harvard Business Review, July/August, pág. 78-91.

HAMEL, G. Y PRAHALAD, C.K. (1993). "Strategy as Stretch and Leverage." Harvard Business Review, vol. 71, no2, March/April, pág. 75-84.

LATORRE, A., DEL RINCÓN, D. Y ARNAL, J. (2003). Bases metodológicas de la investigación educativa. Barcelona: Ediciones Experiencia S.L.

LEAVY, B. (1998). "The Concept of Learning in the Strategy Field". Management Learning, vol. 29, no4, pág. 447-466.

LEONARD BARTON, D. (1995). Wellsprings of Knowledege: Building and Sustaining the Sources of Innovation: Harvard Business School Press.

LOHR, S. L. (1999). Muestreo: Diseño y Análisis. International. Madrid, España: Thomson Editores. 
MATOS, H. (2005). Transformación de la demanda turística. CD - Rom Complete su Conocimiento. Universidad de Matanzas.

MARTÍNEZ, C. (2008). Consideraciones sobre Inteligencia Emocional. La Habana: Editorial Científico - Técnica.

NONAKA, I Y TAKEUCHI, K (1995). The Knowlegge-Creating Company: How Japanese Companies Create in the dynamics of Innovations. New York: Oxford University Press.

PRIETO, I. M (2003). “Una valorización de la gestión del conocimiento para el desarrollo de la capacidad de aprendizaje en las organizaciones: propuesta de un modelo integrador". Universidad de Valladolid, España: Tesis del doctorado, pág 28.

RANT, M. (2001). "The Role of Organization in Knowledge Management: The Empirical Study of the Organization as the Focal Knowledge Creation and Sharing Mechanism." Second European Conference on Knowledge Management. Bled, Slovenia.

RODRÍGUEZ, J.M; MORCILLO, P; CASANI, F. Y RODRÍGUEZ, J. (2001). “Propuesta de un nuevo modelo de estructura organizativa ante el reto de la nueva economía: las estructuras hipertrébol". Comunicación presentada al XI Congreso AECA, Madrid

RODRÍGUEZ, I., SAN MARTÍN, H. (2008). "La satisfacción del turista. Un modelo cognitivoafectivo". Annals of Tourism Research en Español, 122-141

SENGE, P.; ROSS, R.; SMITH, B. ROBERTS, CH. Y KLEINER, A. (1995). La quinta disciplina en la práctica. Estrategias y herramientas para construir la organización abierta al aprendizaje. España: Juan Granica S.A.

STANKEVIVIUTE, J. (2001). "Different Epistemological Perspectives and Their Implications for Knowledge Management in Organizations." Second European Coference on Knowledge Management. Bled, Slovenia.

VALHONDO, D. (2003) "Gestión del conocimiento. Del mito a la realidad". Madrid España: Ediciones Díaz de Santos.

VERA, D. Y CROSSAN, M. (2000). “Organizational Learning, Knowledge Management, and Intellectual Capital: An Integrative Conceptual Model". Working Paper.

WALSH, J. Y UNGSON, G. (1991). "Organizational Memory". Academy of Management Review, vol. 16, no1.

ZACK, M.H. (1999a). "Developing a Knowledge Strategy". California Management Review, vol. 41, no3, pág. 125-145. 